\title{
Development of nuclear emulsion with thick type plastic base for improvement of angular accuracy
}

\author{
MANABE Yuta ${ }^{1}$ \\ Nagoya University \\ Furo-cho, Chikusa-ku, Nagoya, 464-8602, Japan \\ F-lab, Graduate School of Science, Nagoya University \\ E-mail:manabe@flab.phys.nagoya-u.ac.jp
}

MORISHIMA Kunihiro, KITAGAWA Nobuko, NISHIO Akira, KUNO Mitsuaki, MOTO Masaki

Nagoya University

Furo-cho, Chikusa-ku, Nagoya, 464-8602, Japan

F-lab, Graduate School of Science, Nagoya University

E-mail: morishima, kitagawa,nishio,kuno,moto@flab.phys.nagoya-u.ac.jp

Muon radiography is nondestruction research technic for large structure like a pyramid, volcano, blast furnace. The technology measures the density length (=material density $\times$ path length) by calculating penetration rate of muon flux. The nuclear emulsion that is made in Nagoya university detects number of muon tracks and angles. We tried to change its "plastic layer" to thick type for improvement of angular accuracy and estimated "long term performance" and "angular accuracy". In conclusion, the candidate has same long term quality and higher angular resolution.

The 3rd International Symposium on "Quest for the Origin of Particles and the Universe"

5-7 January 2017

Nagoya University, Japan

\section{${ }^{1}$ Speaker}




\section{1. Introduction}

We are doing muon radiography using nuclear emulsion. Muon radiography is similar principal to X-ray radiography, nondestructive inspection for large structure, measure muons flux which penetrate structure and calculate energy loss by comparing free sky flux to inverse calculate substance quantity.

\subsection{Nuclear emulsion}

The nuclear emulsion has structures which $70 \mu \mathrm{m}$ emulsion layer are coated both side of 175 $\mu$ m Poly styren (=PS). This emulsion layers are made by $200 \mathrm{~nm} \mathrm{AgBr}$ crystals and gelatin. We can take pictures of about $1 \mu \mathrm{m} \mathrm{Ag}$ grain by microscope after chareged particles penetrate $\mathrm{AgBr}$ crystals and develop nuclear emulsions.

\subsection{Emulison cloud chamber}

The emulsion cloud chamber $(=\mathrm{ECC})$ is made by stacking alternaately nuclear emulsions and materials like a lead.This detector can measure particle's momentum by measuring multiple coulomb scattering. We have to separate particles by momentum to reject background like low energy muons and electrons in muon radiography especially like a bolcano. Detector's angular accuracy is better, we can measure more detailed scattering angle, so we want improve angular accuracy.

\subsection{Motibasion}

When we detect tracks in nuclear emulsion, at first, take 16 slice pictures each emulison layers. Second, decide particles straigtness and pulse hight by using number of Ag grain existing layer in 16 layers and reconstructe microtrack which is particle track in each emulsion layers. Finaly, reconstruct base track by connecting both side micro tracks to correct gelatin distortion. In this time, $\delta \mathrm{x}$ and $\delta \mathrm{y}$ are microtrack's position accuracy and $\mathrm{L}$ is distance of each emulsion layer, so we want use more thick type plastic base because more distance between both layers provides better angular accuracy.

$$
\delta \tan \theta_{x}=\frac{\sqrt{2}}{L} \sqrt{\delta x^{2}+\delta z^{2} \times\left(\tan \theta_{x}\right)^{2}}
$$

And at the point of flattness, PS base is not good $(175 \mu \mathrm{m} 2.1 \%)$ so we want change it too.

\section{Cycloolefin polymer base test}

This time, we tested $500 \mu \mathrm{m}$ cycloolefin polymer $(=\mathrm{COP})$ for the new base material. It has good flatness $(500 \mu \mathrm{m} \pm 0.88 \%)$

\subsection{Long term test}

When we contact never used material with emulsion, we have to check it doesn't have bad effect on emulsion. (Ex: Contacting silicone rubber increases emulsion's nois density.) We evaluated in accelerated test and real environment test about "fading effect" and "fog up effect". 
Charged particles tracks become thinner by the time between penetrating and developping, it's called "fading effect". It's accelerated in high humidity and high temperature. Developed Ag grain which unrelated charged particle(=fog) increase chronologically, it's called "fog up effect". It's accelerated in low humidity and high temperature.

In this paragraph, "Grain Density (=GD)" is defined as number of grain / 100 $\mu$ m and "Fog Density (=FD)" is defined number of grain / $1000 \mu \mathrm{m}^{3}$. GD means track's probability and FD means emulsion's nois density. The upper graphs are tests about fading effect. Left is accelerating test at temperature is $32^{\circ} \mathrm{C}$ and humidity is $60 \%$ enviromment. Horizontal axis is time, vertical axis is GD. There is no difference betttween PS base emulsion and COP base emulsion in the range of error. Right is real enviromment test, we overlay PS and COP emulsion and put it in real reserch enviromment (Egyptian pyramid, $30^{\circ} \mathrm{C} 30 \%, 49$ day). Horizontal axis is pulse hight $(=\mathrm{PH})$ of track's in COP emulsion, vertical axis is corresponding track's PH and color range is entry of tracks. We can see no difference of base effect. Bottom graphs are tests about fog up effect. Left is accelerating test at temperature is $30 \sim 40^{\circ} \mathrm{C}$ and humidity is $30 \%$ enviromment. Horizontal axis is time, vertical axis is GD. PS emulsion is $25 \times 30 \mathrm{~cm}^{2}$, COP emulison is $12.5 \times 20 \mathrm{~cm}^{2}$ because of base sample size. Fog up effect related to film size too, so we can say only COP is not worse than PS in this condition. Right is real enviromment test same as previous condition, horizontal axis is base type and virtical axis is FD. There is no differnce.

\section{Accelerating test}
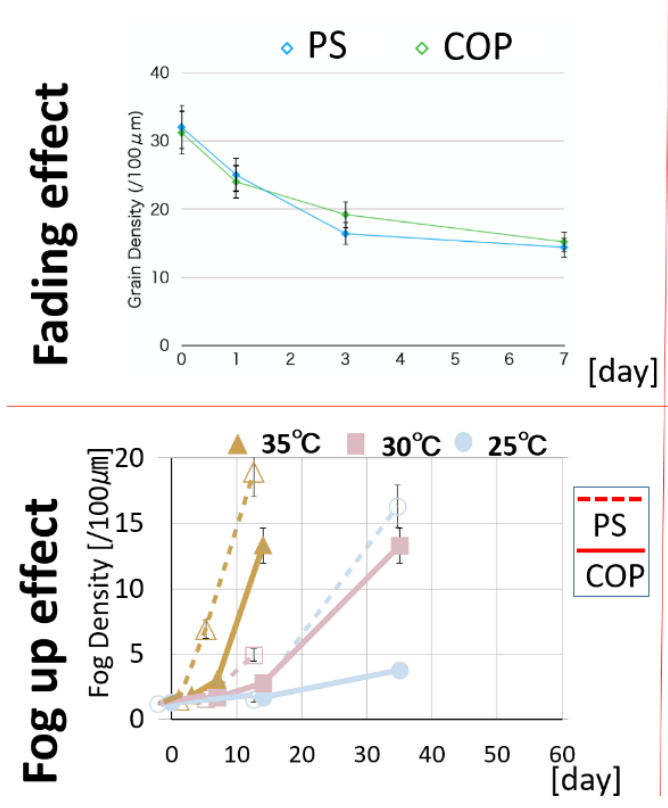

\section{Real environment test}
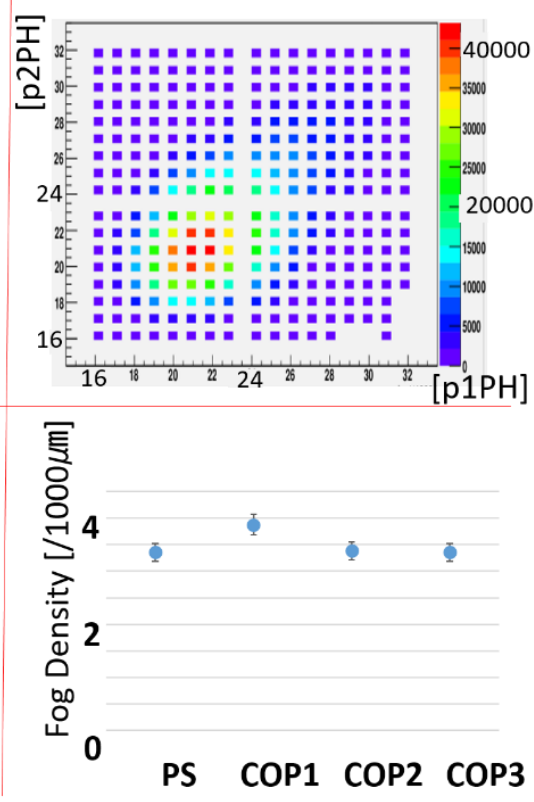

Fig.1 Accelerating and real enviromment test of "Fading effect" and "Fog up effect" results

\subsection{Angular accuracy test}

We exposed $10 \mathrm{GeV}$ pion beam at CERN to measure PS and COP base emulsion's angular accuracy. We stack same type base emulsion and expose beam virtically. Expose angle is $\tan \theta x=$ $0.0,0.2,0.4,0.6,0.8,1.0$ and $\tan \theta y=0.0$. We define angular accuracy that histgram's $1 \sigma$ of (plate $1 \tan \theta-$ plate $2 \tan \theta$ ) about penetrating same track. 


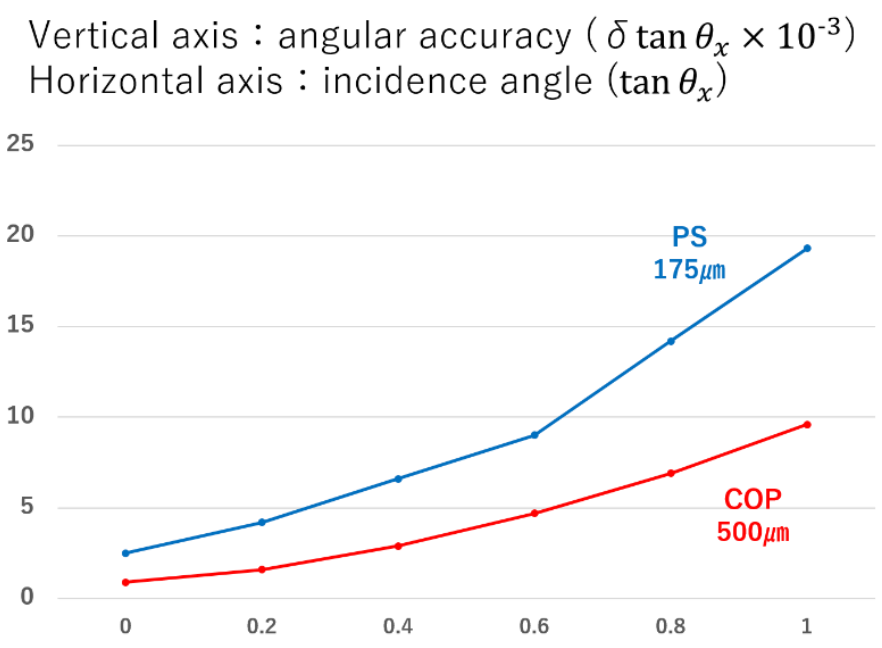

Fig. 2 Angular accuracy test

In this graph, COP base emulsion's angular accracy is about 2 times better than PS base emulsion. We can say changing thick type base is certainly good for angular accuracy but have to estimate this number and connecting rate.

\section{Conclusion}

We checked long term performance and angular accuracy of $175 \mu \mathrm{m}$ PS and $500 \mu \mathrm{m}$ COP base emulsion for muon radiography. The new candidate has same long term quality and higher angular accuracy. We have to estimate this value but can't get other thick base be made of COP now, so we are trying to use other materials like a PMMA to value base's thickness effect. 